\title{
Histone methyltransferase EHMT2 degradation by propionate derived from Bacteroides thetaiotaomicron induced colon cancer apoptosis via epigenetic regulation of TNFAIP1
}

\section{Tae Young Ryu}

Korea Research Institute of Bioscience and Biotechnology

Kwangho Kim

kribb

Tae-Su Han

Korea Research Institute of Bioscience and Biotechnology

Mi-Ok Lee

Korea Research Institute of Bioscience and Biotechnology Jinkwon Lee

Korea Research Institute of Bioscience and Biotechnology Jinhyeon Choi

Korea Research Institute of Bioscience and Biotechnology

Eun-Jeong Jeong

Korea Research Institute of Bioscience and Biotechnology

\section{Da Mi An}

Korea Research Institute of Bioscience and Biotechnology

Cho-Rok Jung

Korea Research Institute of Bioscience and Biotechnology

Jaeeun Jung

Korea Research Institute of Bioscience and Biotechnology

\section{Kunhyang Park}

Korea Research Institute of Bioscience and Biotechnology

Doo-Sang Park

Korea Research Institute of Bioscience and Biotechnology

Dae-Soo Kim

Korea Research Institute of Bioscience and Biotechnology

Mi-Young Son

Korea Research Institute of Bioscience and Biotechnology Hyun-Soo Cho ( $\square$ chohs@kribb.re.kr)

Korea Research Institute of Bioscience and Biotechnology 


\section{Research}

Keywords: B. thetaiotaomicron, propionate, EHMT2, CRC apoptosis, degradation

Posted Date: April 21st, 2020

DOI: https://doi.org/10.21203/rs.3.rs-22596/v1

License: (c) (1) This work is licensed under a Creative Commons Attribution 4.0 International License. Read Full License 


\section{Abstract}

\section{Background}

Bacteroides thetaiotaomicron (BT) is the second most commonly isolated bacterium in the human microbiome after $B$. fragilis and is involved in the hydrolysis of glycosidic bonds in dietary carbohydrates. Although the human microbiome has recently been shown to affect colorectal cancer (CRC) treatment, the mode of action (MOA) between the microbiome and CRC is still unclear.

\section{Methods}

To assess the colon cancer apoptosis, we performed western blot, qRT-PCR and FACS analysis using BT supernatant (Sup), sodium propionate (SP), and EHMT2 specific siRNA. Using RNA-seq analysis and ChIP-seq analysis, we identified HECTD2 (E3 ligase) and TNFAIP1 (EHMT2 target) genes. The antitumor activity of EHMT2 was determined by an in vivo xenograft model.

\section{Results}

We selected propionate derived from BT and showed that it suppressed CRC growth by promoting proteasomal degradation of EHMT2 through HECTD2 upregulation. Moreover, downregulation of EHMT2 reduced the level of $\mathrm{H} 3 \mathrm{~K} 9 \mathrm{me} 2$ on the promoter region of TNFAIP1, and subsequently, upregulation of TNFAIP1 induced apoptosis of CRC cells. Finally, we performed an in vivo study using BIX01294 to inactivate EHMT2 and observed a reduction in the tumor size of the CRC xenograft models.

\section{Conclusions}

We suggest anticancer effects of BT and EHMT2 as therapeutic targets for colon cancer treatment, and we will provide the possibility for synergistic effects of an EHMT2 inhibitor and BT in CRC treatment.

\section{*Authors share co-first authorship}

\section{Background}

Colorectal cancer (CRC) is a major cancer worldwide. Although chemotherapy (5-fluorouracil (5-FU) and oxaliplatin) and targeted therapy (cetuximab and bevacizumab) are used to treat this disease, new therapeutic methods are needed to reduce the side effects and increase the success rate of CRC treatment [1-3]. Recently, the human microbiome, including metabolites and microbiota, has been studied to identify alternative new CRC treatments [4-6].

In the human body, the microbiota mainly colonizes the large intestine and promotes food digestion, regulation of the human immune system, production of vitamins (B12, K, riboflavin), and protection against disease-related bacteria [7-9]. In the colon, the Bacteroides genus is a major group of the human microbiome, and Bacteroides thetaiotaomicron (BT) is the second most commonly isolated bacteria after 
$B$. fragilis [10-12]. BT is a gram-negative obligate anaerobe. This bacterium is involved in polysaccharide metabolism, such as hydrolysis of glycosidic bonds in dietary carbohydrates [10]. In colon cancer, the major bacterium $B$. fragilis showed suppression of inflammation-associated CRC development via polysaccharide A production [13]. In other words, B. fragilis-derived toxins induced CRC development [14]. Although $\mathrm{BT}$ is the second most commonly isolated bacterium in the human microbiome, the relationship between BT and CRC is still unclear. Thus, we examined the anticancer effects of BT using CRC cell lines and elucidated the molecular mechanism of BT-induced apoptosis of CRC cells via epigenetic analysis.

Short-chain fatty acids (SCFAs; butyrate, acetate and propionate) are metabolic products produced via dietary fiber fermentation by the microbiome in the colon [15] and are involved in insulin resistance and colonic inflammation [16]. In colon cancer, SCFAs suppressed CRC growth and metastasis via the induction of hyperacetylation $[17,18]$, and in vivo, SCFAs induced COX-2 expression to suppress azoxymethane/dextran sodium sulfate (AOM/DSS)-induced tumors [19]. Recently, we reported that propionate suppressed CRC growth via downregulation of PRMT1 expression [20]. However, the mode of action (MOA) of propionate for CRC suppression is not yet fully understood.

In colon cancer, the regulation of histone methylation and demethylation is an essential process for the regulation of tumor suppressor and oncogene expression [21-23]. Several histone methyltransferases and demethylases are overexpressed and involved in CRC growth and metastasis via epigenetic regulation [24, 25]. Among them, euchromatic histone-lysine N-methyltransferase 2 (EHMT2) carried out mono- and dimethylation of histone $\mathrm{H} 3$ lysine 9 to generate heterochromatin for the repression of tumor suppressor genes. Knockdown of EHMT2 by siRNA resulted in cell apoptosis, cell cycle arrest, and metastasis in several types of cancer [26-28]. Although EHMT2 is an important therapeutic target for CRC treatment, the regulation of EHMT2 expression in CRC, especially the relationship between EHMT2 and the human microbiome, remains elusive.

Thus, in this study, we demonstrated the anticancer effect of BT supernatant (Sup) at the epigenetic level. Propionate in BT Sup upregulated HECT domain E3 ubiquitin protein ligase 2 (HECTD2) expression to promote proteasomal degradation of EHMT2 via post-translational modifications. Subsequently, downregulation of EHMT2 by propionate induced tumor necrosis factor a-induced protein 1 (TNFAIP1) expression through reduction of the $\mathrm{H} 3 \mathrm{~K} 9 \mathrm{me} 2$ level to promote colon cancer apoptosis. Finally, in an in vivo study, we identified EHMT2 as a therapeutic target for colon cancer treatment with propionate or BT.

\section{Methods}

\section{Bacterial culture}

Bacteroides thetaiotaomicron DS1880, which was isolated from human feces, was cultivated in tryptic soy broth (BD, Sparks, MD, USA) with $5 \%$ horse blood under anaerobic conditions at $37^{\circ} \mathrm{C}$ for $36 \mathrm{~h}$. The bacterial culture was incubated at $65^{\circ} \mathrm{C}$ for $30 \mathrm{~min}$ and centrifuged at $3,000 \mathrm{~g}$ for $10 \mathrm{~min}$. The supernatant was collected in a new tube and kept at $-70^{\circ} \mathrm{C}$ until use. 


\section{Cell Culture And Reagents}

The human CRC cell lines HCT116 and LS174T were purchased from the Korean Cell Line Bank and cultured in RPMI-1640 medium supplemented with 10\% fetal bovine serum (FBS) and 1\% penicillin/streptomycin in a humidified atmosphere with $5 \% \mathrm{CO}_{2}$ at $37^{\circ} \mathrm{C}$. Sodium propionate (SP; P5436) was purchased from Sigma-Aldrich. MG132 (M7449) and cycloheximide (CHX; C4859) were purchased from Sigma-Aldrich. BIX01294 (ab141407) was purchased from Abcam.

\section{Cell Viability Assay}

Cell Counting Kit-8 (CCK8; Dojindo Laboratories) was used to conduct the cell viability assays. Cells were seeded in 6-well plates at $4 \times 105$ cells/well and incubated for $24 \mathrm{~h}$. After BT sup treatment, SP treatment or siRNA transfection for $48 \mathrm{~h}$ or BIX01294 treatment for $24 \mathrm{~h}$, CCK8 solution and RPMI-1640 medium with $10 \%$ FBS were added to each well and incubated with $5 \% \mathrm{CO} 2$ at $37^{\circ} \mathrm{C}$ for $2 \mathrm{~min}$ or $5 \mathrm{~min}$. The absorbance was assessed using a microplate reader at $450 \mathrm{~nm}$. For crystal violet staining, the cells were fixed with methanol for 5 min and stained with $0.1 \%$ crystal violet after BT sup treatment, SP treatment, siRNA infection for $48 \mathrm{~h}$ or BIX treatment for $24 \mathrm{~h}$.

\section{Fluorescence-activated Cell Sorting (facs) Analysis}

After treatment with BT sup, SP, knockdown of EHMT2 for $48 \mathrm{~h}$ or BIX for $24 \mathrm{~h}$, the cells were collected and incubated with the Muse Annexin V and Dead Cell Assay kit (MCH100105; Merck) for 20 min at room temperature. For analysis with the Muse ${ }^{\mathrm{TM}}$ Caspase-3/7 Kit (MCH100108; Merck), the cells were incubated with caspase $3 / 7$ reagent (Merck) for $30 \mathrm{~min}$ in a humidified atmosphere with $5 \% \mathrm{CO} 2$ at $37^{\circ} \mathrm{C}$. After incubation, the cells were incubated with Caspase 7-AAD (Merck) for $5 \mathrm{~min}$ at room temperature. After incubation, $\sim 5 \times 104$ cells were analyzed using a Muse Cell analyzer (Merck). The FACS results were analyzed using Muse 1.6 Analysis software (Merck).

\section{Sirna Transfection}

siRNA duplexes against EHMT2 (siEHMT2; 5'-GCAAAUAUUUCACCUGCCATT-3', 5'UGGCAGGUGAAAUAUUUGCTT-3'), TNFAIP1 (siTNFAIP1; 5'- CUCUCAGGUCAGG UACCUUTT-3', 5'AAGGUACCUGACCUGAGAGTT-3'), and HECTD2 (siHECTD2; 5'-CAUUGAAGACUCUGGGAUUTT-3', 5'AAUCCCAGAGUCUUCAAUGTT-3') were purchased from Bioneer Co., Ltd. Negative control siRNA (siCont; 5'-AUGAACGUGAAU UGCUCAATT-3', 5'-UUGAGCAAUUCACGUUCACTT-3') was used as a control. The siRNAs (100 nM) were transfected into cancer cell lines using RNAiMax (Invitrogen) for $48 \mathrm{~h}$ [29]. 


\section{Semiquantitative Reverse Transcription Pcr And Quantitative Real-time Pcr}

Total RNA was isolated from the indicated cell lines using a Qiagen RNeasy Mini kit (Qiagen) according to the manufacturer's instructions. RNA aliquots of $1 \mu \mathrm{g}$ were then reverse-transcribed using the iScript ${ }^{\text {TM }}$ cDNA synthesis kit (Bio-Rad) according to the standard protocols provided by the manufacturer. For semiquantitative RT-PCR, CDNA was used as a template for PCR using AccuPower ${ }^{\circledR}$ HotStart PCR PreMix (Bioneer). Quantitative RT-PCR (EHMT2: annealing temperature $55^{\circ} \mathrm{C}, 35$ cycles; ACTB: annealing temperature $58^{\circ} \mathrm{C}, 28$ cycles) was performed using the SimpliAmp Thermal Cycler (Applied Biosystems) following the manufacturer's instructions. Quantitative real-time PCR was performed on CDNA samples using Brilliant III Ultra-Fast SYBR® Green QPCR Master Mix, and the signal was detected using the AriaMx Real-Time PCR System (both from Agilent Technologies). The fluorescence threshold value was calculated using Agilent Aria 1.6 software (Agilent Technologies) $(20,21)$. The PCR primers were as follows: EHMT2 forward, 5'-GGGCTACTGCCTCTTCTACG AGTC-3' and reverse, 5'GTCTTTGTACTGCCGGTCCT CGTAG-3'; TNFAIP1 forward, 5'- CATCACATCCCTAAAGGAGGAGG-3' and reverse, 5'-AGCAGGTGGTCGTCAGAGTT-3'; HECTD2 forward, 5'-ATTCGTTTCCCTCCCTGCTG-3' and reverse, 5'-AGTTCACC TGCACATCTTGTTT-3'; and ACTB forward, 5'-ACTCTTCCAGCCTTCCTTCC-3' and reverse, 5'-CAATGCCAGGGTACATGGTG-3'.

\section{Western Blot Analysis}

The cells were washed once with phosphate-buffered saline (PBS) and then lysed in cold lysis buffer (50 mM Tris-HCl, pH 7.4, 150 mM NaCl, 1\% Triton X-100, 0.1\% SDS, 1 mM EDTA, 1 mM Na3VO4, 1 mM $\mathrm{NaF}$ and $1 \mathrm{X}$ protease inhibitor cocktail). Cell lysates were centrifuged at $14,000 \mathrm{xg}$ for $20 \mathrm{~min}$ at $4^{\circ} \mathrm{C}$ and then boiled in 5X sample buffer following protein determination (BSA, 23208; Thermo Fisher Scientific). The protein samples were subjected to western blot analysis. For western blot analysis, nitrocellulose membranes (1620145; Bio-Rad ), blocking reagent (5\% skim milk, $1 \mathrm{~h}$ at room temperature), and 4-20\% precast gels (456-1094; Bio-Rad) were used with the indicated antibodies at a 1:1,000 dilution ratio (22). The samples were stained with the following antibodies: EHMT2 (ab185050) and TNFAIP1 (ab86934) from Abcam, PARP (9542) from Cell Signaling Technology, FLAG (F1804) from Sigma-Aldrich, and HA (sc-805) and ACTB (sc-47778) from Santa Cruz Biotechnology. Secondary antibodies (rabbit; sc-2357, mouse; sc-2031; Santa Cruz Biotechnology) were incubated at room temperature for $1 \mathrm{~h}$, and ECL solution (170-5060; Bio-Rad) was used for visualization. A chemiluminescence imaging system (Mini HD9; UVitec) was used for imaging.

\section{Immunoprecipitation}

pCAGGS-n3FC (Mock), pCAGGS-n3FCEHMT2 (3 × FLAG-EHMT2), and Ub-HA were transfected into HCT116 cell lines using FuGENE® 6 Transfection Reagent (Promega) for 48 h. Transfected HCT116 cells 
were lysed with cold lysis buffer (50 mM Tris-HCl, pH 7.4, $150 \mathrm{mM} \mathrm{NaCl}, 1 \%$ Triton X-100, 0.1\% SDS, $1 \mathrm{mM}$ EDTA, $1 \mathrm{mM} \mathrm{Na}$ VO 4, $1 \mathrm{mM} \mathrm{NaF}$ and 1X protease inhibitor cocktail). In a typical immunoprecipitation reaction, $800 \mu \mathrm{g}$ of whole-cell extract was incubated with an optimum concentration of primary antibody and ANTI-FLAG ${ }^{\circledR}$ M2 Affinity Gel (A2220; Sigma-Aldrich). After the beads had been washed three times in $1 \mathrm{ml}$ of Tris-buffered saline ( $\mathrm{pH}$ 7.6), proteins that bound to the beads were eluted by boiling in $2 \mathrm{X}$ sample buffer (Thermo Scientific).

\section{Chromatin Immunoprecipitation}

ChIP was performed with Magna ChIP A/G (Magna0013 and Magna0014; Millipore) following the manufacturers' instructions. HCT116 cells were transfected with siCont and siEHMT2 for $48 \mathrm{~h}$ or treated DMSO and BIX for $24 \mathrm{~h}$ crosslinked with $1 \%$ formaldehyde (Sigma-Aldrich) for $10 \mathrm{~min}$ at room temperature, and quenched with $1 \mathrm{X}$ glycine (Millipore) for $5 \mathrm{~min}$ at room temperature. Then, the HCT116 cells were washed with cold 1X PBS (containing 1X Protease inhibitor Cocktail $₫)$. After nuclear extraction, the chromatin solution was sonicated using a Bioruptor ${ }^{\circledR}$ Pico sonication device (B01060010; Diagenode) with 15 cycles of 30 seconds $\mathrm{ON}$ and 30 seconds OFF to obtain 200-1000 bp chromatin fragments. Sheared chromatin was incubated with $2 \mu \mathrm{g}$ of H3K9me2 (ab1220; Abcam) antibody or $2 \mu \mathrm{g}$ of H3K9ac (ab4441; Abcam) antibody with $20 \mu$ l of Magna ChIP A/G magnetic beads (Millipore) overnight at $4{ }^{\circ} \mathrm{C}$. The complexes were incubated with ChIP elution buffer and RNase A mixture for $30 \mathrm{~min}$ at $37^{\circ} \mathrm{C}$ and then incubated with proteinase $\mathrm{K}$ for $2 \mathrm{~h}$ at $62^{\circ} \mathrm{C}$. After DNA purification using spin columns, the samples were analyzed by semiquantitative PCR using TNFAIP1 Primer. The primers were as follows: TNFAIP1 P1 forward 5'-CTGGCAGCCGAACACAAGT-3', and reverse, 5'-CCAA GCCAGATTCATGGGAGT-3' ,TNFAIP1 P2 forward 5'-ACTCCCATGAATCTGGCTTGG-3', and reverse ,5'-GCTCAGATGCTCAGACACGC-3'.

\section{Immunocytochemistry}

Cultured cells were fixed in $4 \%$ paraformaldehyde at room temperature for $10 \mathrm{~min}$, permeabilized in $0.5 \%$ Triton X-100 (Sigma-Aldrich) in PBS for 10 min and blocked with $5 \%$ bovine serum albumin in PBS for $30 \mathrm{~min}$. Fixed cells were incubated with anti-EHMT2 antibody (ab185050; Abcam) and anti-TNFAIP1 antibody (ab86934; Abcam) overnight at $4{ }^{\circ} \mathrm{C}$ and stained with Alexa Fluor-conjugated secondary antibodies (Life Technologies). Fluorescent images were obtained using a CELENA® S Digital Imaging System (Logos Biosystems).

\section{Immunohistochemistry}

Paraffin-embedded sections of colon tumor tissue array (T8235722-2, Biochain) were processed in a microwave $\left(90^{\circ} \mathrm{C}\right.$ ) with antigen-retrieval solution ( $\mathrm{pH}$ 9) (S2367; Dako), treated with a peroxidase-blocking reagent, and then treated with a protein-blocking reagent (K130, X0909; Dako). Tissue sections were incubated with rabbit anti-EHMT2 antibody (68851; CST) and rabbit anti-TNFAIP1 antibody (ab86934; 
abcam) followed by incubation with an HRP-conjugated secondary antibody (Dako).

Immunoreactivitywas visualized with a chromogenic substrate (Liquid DAB Chromogen; Dako). Finally, tissue specimens were stained with Mayer's hematoxylin solution (Hematoxylin QS; Vector Laboratories) for 5 seconds to discriminate the nucleus from the cytoplasm.

\section{Rna-seq And Analysis}

Using TruSeq RNA Sample Preparation Kit V2, purification and library construction were carried out with total RNA, and Illumina HiSeq 2500 machines (Illumina, San Diego, CA, USA) were used for sequencing with a read length of $2 \times 100$ bases. FastQC v.0.11.4 was used for the quality of the paired-end reads. Cutadapt v.1.15 and Sickle v. 1.33 was used for filtering low-quality reads and adaptors. Cufflinks version 2.2.1 was used for calculation of FPKM (fragments per kilobase of transcripts per million mapped reads) values. Cuffdiff was used to select differentially expressed genes (DEGs) (fold change $>2$ ). All Gene Ontology and KEGG pathway enrichment analyses were performed with DAVID ver. 6.8 and CluGO ver. 2.5.5 in Cytoscape ver. 3.7.1. GSEA analysis was performed with GSEA ver. 4.0.1.

\section{Mouse Experiment}

To establish a xenograft mouse model, HCT116 cells were implanted into the flanks of six-week-old Balb/c-nu female mice (Orient Bio. Sungnama, Korea). BIX01294 was intraperitoneally injected into mice every three times a week. Tumor size was measured twice every week, and the tumor volume was calculated by length $(L) \times$ width $(W) \times$ height $(H)$. On day 23 , all mice were sacrificed. The animal experiments were approved by the Committee on Animal Experimentation of the Korea Research Institute of Bioscience and Biotechnology.

\section{Statistical analysis}

The results are expressed as the mean \pm SD (error bars) of three independent experiments. One-way analysis of variance (ANOVA) with the Bonferroni post hoc test was performed using GraphPad Prism 5.0 (GraphPad Software, Inc., La Jolla, CA, USA). The cut-off for significance was $p<0.05$.

\section{Results}

\section{BT Sup culture medium suppressed the growth of HCT116 cells}

In the human gut flora, BT is one of the most common bacteria and is the second most isolated strain after $B$. fragilis [10-12]. To evaluate the anticancer effect of BT in colon cancer, we collected BT Sup and added it to the culture medium of CRC cell lines. As shown in Fig. 1a and b, cell growth in the BT Sup treatment group was significantly inhibited compared to that in the control group (media only), as 
demonstrated by crystal violet (CV) staining and Cell Counting Kit-8 (CCK8) assays. In addition, as shown by FACS analysis of caspase 3/7 activity, BT Sup treatment induced caspase 3/7 activity (Fig. 1c). Western blot analysis indicated that cleaved PARP was increased by BT Sup treatment, implying that anticancer factors derived from BT may suppress CRC cell line growth via induction of cell apoptosis (Fig. 1d). Next, to identify the anticancer factors in BT Sup, we performed HPLC analysis and detected the SCFAs. Finally, among the list of metabolites, propionate $(2.172 \mathrm{~g} / \mathrm{l})$ was selected for further analysis of its anticancer effect on colon cancer (Supplementary Fig. S1).

\section{Propionate treatment induced cell apoptosis in HCT116 and LS174T cells}

To assess the anticancer effects of propionate in colon cancer, we performed a cell growth assay with HCT116 and LS174T cells after treatment with sodium propionate (SP). As shown by CV staining and CCK8 assays, cell growth was significantly suppressed in the SP treatment groups compared to the negative control groups (Fig. 1e and f). Moreover, western blot analysis demonstrated that the levels of cleaved PARP were increased in HCT116 and LS174T cells treated with SP (Fig. 1g). In addition, we performed FACS analysis to measure caspase $3 / 7$ activity after SP treatment and observed the induction of caspase 3/7 activity in CRC cell lines after treatment with SP (Fig. 1h). Additionally, SP treatment clearly induced early and late apoptosis, as shown by FACS analysis (Fig. 1i). In RNA-seq analysis of HCT 116 cells treated with SP, 398 upregulated genes and 474 downregulated genes were detected (over 2-fold). Using differentially expressed genes (DEGs), we performed Gene Ontology (GO) analysis using the Database for Annotation, Visualization and Integrated Discovery (DAVID) and found enrichment of negative regulation of growth, positive regulation of apoptosis, cell adhesion, and inflammatory response (Fig. 1j). Additionally, in GSEA (gene set enrichment analysis), the regulation of apoptosis was regulated by SP treatment compared to the control group (Fig. $1 \mathrm{k}$ ). Thus, these findings suggested that treatment with propionate derived from BT induced apoptosis of CRC cell lines.

\section{EHMT2 is a target for propionate-induced cell apoptosis in colon cancer}

Next, to identify the effect of cell apoptosis induced by SP treatment, we focused on histone lysine methyltransferases (HMTs) and demethylases (HDLs) because several types of HMTs and HDLs are overexpressed in CRC, and knockdown of HMTs and HDLs suppresses CRC proliferation via apoptosis of CRC cells [24, 25]. Using in silico epigenetic panels, we selected overexpression of HMTs and HDLs (1.5fold, colon cancer/normal) with 521 colon cancer and 51 normal samples derived from TCGA data portal (https://tcga-data.nci.nih.gov/). We selected EHMT2 as the effector gene of SP-induced apoptosis in CRC (Fig. 2a). In histochemical analysis using a CRC tissue microarray, the expression level of EHMT2 was induced in the CRC tissues compared to the normal placenta (Fig. 2b). EHMT2 is an HMT that is involved in H3K9 dimethylation for the formation of heterochromatin in transcriptional gene regulation. Moreover, 
in breast, bladder and lung cancer, knockdown of EHMT2 was shown to be involved in cell apoptosis and cell migration/invasion [26-28,30]. After treatment with SP in the CRC cell lines, we could not detect any changes in EHMT2 at the transcriptional level, but in western blot analysis, the expression of EHMT2 was significantly reduced (Fig. $2 \mathrm{c}$ and d). Additionally, treatment with BT Sup reduced EHMT2 expression at the protein level but not at the transcriptional level (Fig. 2e). In immunocytochemical analysis after treatment with SP or BT Sup, the intensity of EHMT2 in the SP or BT-treated cells was significantly decreased compared to that in the control cells (Fig. $2 f$ and g). Therefore, we hypothesized that the propionate in BT Sup may be involved in translational modification and proteasomal degradation. Next, to verify whether SP treatment induced the proteasomal degradation of EHMT2, we performed western blot analysis after cotreatment with SP and MG132, which is an inhibitor of proteasomal degradation, in HCT116 and LS174T cells. In the cotreatment group, the reduction in EHMT2 expression by SP treatment was recovered by MG132 treatment compared with the control, suggesting that SP induced the proteasomal degradation of EHMT2 (Fig. 2h). Moreover, after treatment with $\mathrm{CHX}$, a protein synthesis inhibitor, we detected an increase in the protein degradation induced by SP treatment compared with that of the control group in the CRC cell lines (Fig. 2i). Finally, to assess the status of EHMT2 polyubiquitination by propionate, we cotransfected FLAG-tagged EHMT2 and HA-tagged ubiquitin into HCT116 cells treated with SP and/or MG132 and performed immunoprecipitation experiments with an anti-FLAG antibody. As shown in Fig. 2j, EHMT2 polyubiquitination in the SP and MG132 treatment groups was significantly induced compared to that in the control groups. Thus, propionate affected EHMT2 stability via regulation of post-translational modification for proteasomal degradation; consequently, the CRC cell lines were suppressed cell proliferation and induced cell apoptosis. This is the first report of a relationship between propionate treatment and translational modification.

\section{Knockdown of EHMT2 was involved in the apoptosis of CRC cells}

As shown by the RNA-seq results after EHMT2 knockdown, the apoptotic process, cell cycle and regulation of the mitotic cell cycle were enriched in $\mathrm{GO}$ analysis compared with those of siCont treatment (Fig. 3a). To assess whether downregulation of EHMT2 by SP treatment induced cell apoptosis, we designed EHMT2-specific siRNA and performed a cell growth assay after treatment with the EHMT2 siRNA. As shown in Fig. 3b and c, cell growth in the CRC cell lines determined by CV staining and CCK8 assays was suppressed by EHMT2 knockdown compared with that in the siCont group, as demonstrated by SP treatment. In western blot analysis, cleaved PARP was increased by EHMT2 knockdown (Fig. 3d). In FACS analysis for caspase 3/7 activity and apoptosis, the EHMT2 knockdown group showed upregulation of caspase $3 / 7$ activity in the CRC cell lines (Fig. 3e). The population of early and late apoptotic cells was increased by EHMT2 knockdown compared to that of the siCont group (Fig. 3f). Thus, we suggest that EHMT2 downregulation by SP treatment suppressed cell proliferation via cell apoptosis in CRC cell lines, indicating that EHMT2 is a key protein for SP-induced cell apoptosis. 


\section{Treatment With Sp Induced Hectd2 Expression To Degrade Ehmt2}

Next, to identify the mechanism for EHMT2 degradation by SP treatment, we reanalyzed the RNA-seq data and found upregulation of E3-ligase expression by SP treatment compared to the control treatment (Fig. 4a). To assess the relationship between proteasomal degradation and E3 ligase, we performed recovery analysis after treatment with siRNA and SP and observed that HECTD2 knockdown helped rescue the degradation of EHMT2 induced by SP treatment (Supplementary Fig. S2). In qRT-PCR, we reconfirmed the upregulation of HECTD2 expression by SP and BT Sup treatment (Fig. 4b and c). HECTD2 is an E3 ligase that is recognized as a substrate for ubiquitin transfer from the E2 ubiquitinconjugating enzyme [31]. In siRNA analysis of HECTD2, the degradation of EHMT2 by SP was inhibited by HECTD2 knockdown (Fig. 4d). In the CHX treatment analysis, the degradation rate of EHMT2 by SP was reduced by siHECTD2 treatment compared to the control siRNA treatment (Fig. 4e). In addition, we clearly detected a reduction in the polyubiquitination status in the siHECTD2 and SP treatment groups (Fig. 4f). Additionally, FACS analysis for caspase 3/7 activity showed that cotreatment with siHECTD2 and SP decreased caspase 3/7 activity compared to that in SP treatment only (Fig. 4g). Thus, the induction of HECTD2 expression by propionate may promote the proteasomal degradation of EHMT2; accordingly, the growth of colon cancer was suppressed via the apoptosis induced by EHMT2 reduction.

\section{TNFAIP1 is a direct target of EHMT2 for the induction of apoptosis of CRC cells}

To identify EHMT2 direct target genes, we performed ChIP-seq analysis after treatment with siEHMT2, and we focused on the TNFAIP1 gene for SP-induced cell apoptosis because H3K9 dimethylation status was reduced in TNFAIP1 promoter regions of siEHMT2 groups compared to that in siCont groups (Fig. 4h). Additionally, to confirm this result, we performed a ChIP assay after treatment with EHMT2 siRNA again. In Fig. 4i, we designed two ChIP primers (P1 and P2) targeting the promoter regions of TNFAIP1 and performed ChIP assays using anti-H3K9me2 and anti-H3K9ac antibodies. H3K9 dimethylation status was significantly decreased in the promoter region of the TNFAIP1 gene after EHMT2 knockdown compared to that in the siCont group. In addition, H3K9 acetylation in the promoter region of the TNFAIP1 gene increased, suggesting that EHMT2 directly regulated TNFAIP1 expression via alteration of chromatin structures (Fig. $4 \mathrm{j}$ and $\mathrm{k}$ ).

\section{Epigenetic regulation of TNFAIP1 by EHMT2 induced the apoptosis of CRC cells}

EHMT2 was involved in the negative regulation of gene expression via the formation of a heterochromatin structure through H3K9 dimethylation [32]. Thus, we selected upregulated genes, which 
are candidate genes for direct regulation by EHMT2, after EHMT2 knockdown. TNFAIP1 is an early response gene induced by IL- 6 and TNFa and is involved in cell apoptosis, DNA repair and DNA synthesis. In gastric cancer, overexpression of TNFAIP1 induced by knockdown of miR-372 promoted cell apoptosis via regulation of the NF-kB signaling pathway. In HeLa cell lines, overexpressed TNFAIP1 also increased cell apoptosis via downregulation of NF-kB expression [33-36]. Thus, we selected TNFAIP1 as the final target of EHMT2-induced apoptosis. In the RNA-seq results, we found upregulation of TNFAIP1 after SP treatment and EHMT2 knockdown in the CRC cell lines, and the induction of TNFAIP1 was confirmed by qRT PCR analysis (Fig. 5a). In addition, treatment with BT Sup increased TNFAIP1 expression compared to that of the control group, implying that propionate induced the expression of TNFAIP1 via downregulation of EHMT2 (Fig. 5b). In immunocytochemical analysis, the intensity of TNFAIP1 increased after BT Sup, SP or siEHMT2 treatment compared to that of the control cells (Fig. 5c). Thus, we suggest that the downregulation of EHMT2 induced TNFAIP1 expression at the transcriptional level. Next, to assess the relationship between TNFAIP1 and EHMT2 knockdown, we performed a cell growth assay with CV staining and CCK8 analysis. As shown in Fig. $5 \mathrm{~d}$ and $5 \mathrm{e}$, growth recovery by cotreatment with EHMT2 and TNFAIP1 siRNAs compared with that of the siEHMT2 treatment only was observed, as demonstrated by $\mathrm{CV}$ staining and CCK8 assays. In western blot analysis, the induction of increased PARP by EHMT2 knockdown was reduced by cotreatment with siEHMT2 and siTNFAIP1 (Fig. 5f). In addition, FACS analysis to estimate caspase 3/7 activation and apoptosis showed that cotreatment with EHMT2 and TNFAIP1 siRNAs decreased caspase $3 / 7$ activity (Fig. $5 \mathrm{~g}$ ). Thus, TNFAIP1 is an effector gene after EHMT2 degradation by SP treatment in CRC cell lines.

Next, to confirm the interaction between EHMT2 and TNFAIP1, we performed correlation analysis using CRC public RNA-seq results derived from TCGA portal. Figure $5 \mathrm{~h}$ shows that EHMT2 and TNFAIP1 exhibited a negative correlation $\left(R^{2}=-0.2, P=0.04\right)$. Additionally, in immunohistochemical analysis with a CRC tissue microarray, the expression of EHMT2 in the tissue microarray was negatively correlated with TNFAIP1 expression (Fig. 5i). Thus, we suggest that SP-induced proteasomal degradation of EHMT2 reduces H3K9 dimethylation in the promoter region of TNFAIP1; consequently, apoptosis of CRC cells is increased by TNFAIP1 upregulation.

\section{Ehmt2 Is A Therapeutic Target For Crc}

Using the RNA-seq results of colon cancer and normal tissues derived from the TCGA portal, we determined the prognostic value of EHMT2 in CRC. The high-expression EHMT2 group showed a poor prognosis compared to the low-expression EHMT2 group ( $p<0.001$; Fig. 6a), indicating that EHMT2 is an attractive therapeutic target for $\mathrm{CRC}$ treatment and can be used for the prevention of CRC incidence. To assess EHMT2 as a therapeutic target of CRC, we used a BIX01294 inhibitor, which is a specific inhibitor of EHMT2 activity [37]. After treatment with BIX01294, the expression of TNFAIP1 significantly increased by reducing EHTM2 activity, as shown by qRT-PCR and immunocytochemical analysis (Fig. 6b and c), and cell growth also decreased, as demonstrated by CV staining (Fig. 6d). In the ChIP assay after treatment with BIX01294, we clearly found a reduction in H3K9me2 status and induction of H3K9ac 
status in the promoter regions of TNFAIP1 (Fig. 6e). Moreover, BIX01294 induced cleaved PARP (western blot analysis) and caspase 3/7 activity (FACS analysis) (Fig. $6 \mathrm{f}$ and g). Finally, the population of early and later apoptotic cells increased after BIX01294 treatment in CRC cell lines (Fig. 6h). To verify the CRC growth inhibitory effect of EHMT2 inactivation in vivo, we performed xenograft analysis with BIX01294 and found that the tumor size was reduced in the BIX01294 treatment group compared with the control group (Fig. 6i-k). Therefore, we suggest that SP can be used for the prevention of CRC incidence and that cotreatment with an EHMT2 inhibitor and that propionate/BT may exhibit a synergistic effect for CRC treatment.

\section{Discussion}

SCFAs such as butyrate and propionate can act as HDAC inhibitors to affect transcriptional regulation of gene expression $[17,18]$. After treatment with SCFAs in CRC, we observed upregulation of apoptosisrelated genes via the induction of histone acetylation on promoter regions, implying that SCFAs are useful anticancer inhibitors. Additionally, the microbiome that produces SCFAs has also been investigated for its effects on health [15]. To date, gene regulation by SCFAs has mainly been mediated at the transcriptional level, but no reports have examined the relationship between post-translational modification and SCFA treatment in colon cancer. Therefore, in this study, we first suggested a novel function of propionate in CRC cell lines, according to the enhancement of EHMT2 proteasomal degradation by propionate in these cells (Fig. 2).

For rapid elimination of proteins in cells, the ubiquitin-proteasome pathway is the most effective process for small peptide recycling during new protein synthesis. In the proteasomal degradation pathway, polyubiquitinated proteins bound to the proteasome by E3 complex proteins are unfolded and degraded. Recently, several papers reported that the proteasome itself has been recognized as a potential therapeutic target in cancer treatment [38]. After cotreatment with SP and MG132 in CRC cell lines, the expression of EHMT2 was rescued from the reduction induced by SP only. In addition, cotreatment with $\mathrm{CHX}$ and SP showed more rapid degradation of EHMT2 expression than SP only treatment, suggesting that SP treatment induced EHMT2 proteasomal degradation (Fig. $2 \mathrm{~h}$ and i). Using RNA-seq and qRT-PCR analysis, we observed upregulation of HECTD2 by SP and BT Sup treatment to promote EHMT2 degradation. Using GENT analysis (http://gent2.appex.kr/gent2/), we found that the expression level of HECTD2 was not different between the control and CRC samples (Supplementary Fig. S3), implying that upregulation of HECTD2 by propionate in CRC promotes EHMT2 proteasomal degradation via induction of polyubiquitination. However, the pathway of HECTD2 upregulation by propionate requires further study.

To assess the anticancer activity of SP in CRC cell lines, we selected the proper concentration of SP ( $2.5 \mathrm{mM} \sim 5 \mathrm{mM}$ ) to perform biochemical analysis and observed SP-induced apoptosis in HCT116 and LS174T cell lines. SCFAs such as propionate, butyrate and acetate are microbial fermentation products generated using dietary fibers in the human colon. In the lumen of the human colon, the concentrations of propionate and acetate are $10 \mathrm{mM} \sim 100 \mathrm{mM}$ [39]; thus, we considered that the concentration of 
propionate used in this study was appropriate to test the anticancer effect of this molecule. Moreover, using human enterocytes that differentiated from human embryonic stem (ES) cells and HCT116 cells, we performed a cell growth assay. In Supplementary Fig. S4, $5 \mathrm{mM}$ propionate did not affect growth inhibition in human enterocytes; in other words, HCT116 was significantly suppressed in the propionate group. Thus, we suggest that propionate derived from the microbiome can prevent colon cancer incidence and can show a synergistic effect with a specific inhibitor for CRC patients. Finally, we also suggested the importance of dietary fiber for the microbiome in the colon.

To date, the clinical application of SP in cancer has not been performed, although SP has shown anticancer effects in several types of cancers, and SCFAs play a critical role in the maintenance of epithelial cells for colonic health [39]. Therefore, although a clinical study with propionate has not been performed, we suggest (1) the intake of the useful microbiota for the production of propionate to inhibit CRC growth and metastasis, (2) the use of dietary fiber for propionate production, and (3) the screening of novel microbiota that produce propionate for analysis of propionate in CRC therapy.

\section{Conclusions}

In summary, the propionate derived from BT in colon cancer decreased the protein stability of EHMT2 by promoting proteasomal degradation. As a result, a reduction in EHMT2 expression also decreased the level of histone H3K9 dimethylation in the promotor region of TNFAIP1. Finally, upregulation of TNFAIP1 by epigenetic regulation suppressed CRC proliferation via the cell apoptosis machinery (Fig. 7). Thus, using the results of this study, we suggest the importance of the microbiome in the colon for the prevention of colon cancer, and SCFAs such as propionate and HMT-specific inhibitors could show synergistic effects for colon cancer treatment. Moreover, dietary therapy for CRC patients is also emphasized to help cure CRC.

\section{Abbreviations}

BT ; Bacteroides thetaiotaomicron, EHMT2; euchromatic histone-lysine N-methyltransferase 2, HECTD2; HECT domain E3 ubiquitin protein ligase 2, TNFAIP1; tumor necrosis factor a-induced protein 1, CRC; colorectal cancer, MOA; mode of action, SP; sodium propionate, Sup; supernatant, 5-FU; 5-fluorouracil, SCFA; short-chain fatty acid, CHX; cycloheximide, CCK8; Cell Counting Kit-8, FACS; fluorescence-activated cell sorting, ChIP; chromatin immunoprecipitation, CV; crystal violet, DEG; differentially expressed genes, GO; Gene Ontology, DAVID; Database for Annotation, Visualization and Integrated Discovery, GSEA; gene set enrichment analysis, HMT; histone methyltransferases, HDM; histone demethylases

\section{Declarations}

Availability of data and materials

All data generated or analyzed during the current study are included in this published article (and its supplementary files) 
Ethics approval and consent to participate

Not applicable

Consent for publication

Not applicate

Competing interests

The authors declare that they have no competing interests.

Funding

This work was supported by a grant from the National Research Foundation of Korea (NRF) grant funded by the Ministry of Science, ICT and Future Planning (2020R1A2B5B01002028 and 2018M3A9H3023077), and the KRIBB Research Initiative Program.

Authors' Contributions

Conception and design: D.-S. Kim, M.-Y. Son, H.-S. Cho; Development of methodology: K. Kim, T.Y Ryu, J. Lee, D.M. An, C.-R. Jung, D.-S. Park ; Animal study: T.-S. Han, E.-J. Jeong, J. Choi; Analysis and interpretation of data: J. Jung, K. Park; Writing and review of manuscript: C.-R. Jung, D.-S. Kim, D.-S. Park, M.-Y. Son, H.-S. Cho; Study supervision: D.-S. Kim, M.-Y. Son, H.-S. Cho

Acknowledgements

Not applicable

\section{References}

1. Gelibter AJ, Caponnetto S, Urbano F, Emiliani A, Scagnoli S, Sirgiovanni G, Napoli VM, Cortesi E. Adjuvant chemotherapy in resected colon cancer: When, how and how long? Surg Oncol. 2019;30:100-7.

2. Kim BJ, Jeong JH, Kim JH, Kim HS, Jang HJ. The role of targeted agents in the adjuvant treatment of colon cancer: a meta-analysis of randomized phase III studies and review. Oncotarget. 2017;8:31112-8.

3. Labianca R, Milesi L, Mosconi S, Pessi MA, Beretta GD, Quadri A. The role of adjuvant chemotherapy in colon cancer. Surg Oncol. 2007;16(Suppl 1):93-6.

4. Leystra AA, Clapper ML. Gut Microbiota Influences Experimental Outcomes in Mouse Models of Colorectal Cancer. Genes (Basel) 2019, 10.

5. Saus E, Iraola-Guzman S, Willis JR, Brunet-Vega A, Gabaldon T. Microbiome and colorectal cancer: Roles in carcinogenesis and clinical potential. Mol Aspects Med. 2019;69:93-106.

6. Yaghoubi A, Khazaei M, Avan A, Hasanian SM, Soleimanpour S. The bacterial instrument as a promising therapy for colon cancer. Int J Colorectal Dis 2020.

7. Aira A, Feher C, Rubio E, Soriano A. The Intestinal Microbiota as a Reservoir and a Therapeutic Target to Fight Multi-Drug-Resistant Bacteria: A Narrative Review of the Literature. Infect Dis Ther. 
2019;8:469-82.

8. Kolodziejczyk AA, Zheng D, Elinav E. Diet-microbiota interactions and personalized nutrition. Nat Rev Microbiol. 2019;17:742-53.

9. LeBlanc JG, Levit R, Savoy de Giori G, de Moreno de LeBlanc A. Application of vitamin-producing lactic acid bacteria to treat intestinal inflammatory diseases. Appl Microbiol Biotechnol 2020.

10. Porter NT, Luis AS, Martens EC. Bacteroides thetaiotaomicron. Trends Microbiol. 2018;26:966-7.

11. Sadarangani SP, Cunningham SA, Jeraldo PR, Wilson JW, Khare R, Patel R. Metronidazole- and carbapenem-resistant bacteroides thetaiotaomicron isolated in Rochester, Minnesota, in 2014. Antimicrob Agents Chemother. 2015;59:4157-61.

12. Snydman DR, Jacobus NV, McDermott LA, Golan Y, Goldstein EJ, Harrell L, Jenkins S, Newton D, Pierson $\mathrm{C}$, Rosenblatt $\mathrm{J}$, et al. Update on resistance of Bacteroides fragilis group and related species with special attention to carbapenems 2006-2009. Anaerobe. 2011;17:147-51.

13. Lee YK, Mehrabian P, Boyajian S, Wu WL, Selicha J, Vonderfecht S, Mazmanian SK: The Protective Role of Bacteroides fragilis in a Murine Model of Colitis-Associated Colorectal Cancer. mSphere $2018,3$.

14. Purcell RV, Pearson J, Aitchison A, Dixon L, Frizelle FA, Keenan JI. Colonization with enterotoxigenic Bacteroides fragilis is associated with early-stage colorectal neoplasia. PLoS One. 2017; 12:e0171602.

15. Tan J, McKenzie C, Potamitis M, Thorburn AN, Mackay CR, Macia L. The role of short-chain fatty acids in health and disease. Adv Immunol. 2014;121:91-119.

16. McNabney SM, Henagan TM. Short Chain Fatty Acids in the Colon and Peripheral Tissues: A Focus on Butyrate, Colon Cancer, Obesity and Insulin Resistance. Nutrients 2017, 9.

17. Sekhavat A, Sun JM, Davie JR. Competitive inhibition of histone deacetylase activity by trichostatin A and butyrate. Biochem Cell Biol. 2007;85:751-8.

18. Davie JR. Inhibition of histone deacetylase activity by butyrate. J Nutr. 2003;133:2485S-2493S.

19. Tian $Y, X u$ Q, Sun L, Ye Y, Ji G. Short-chain fatty acids administration is protective in colitisassociated colorectal cancer development. J Nutr Biochem. 2018;57:103-9.

20. Mun SJ, Ryu JS, Lee MO, Son YS, Oh SJ, Cho HS, Son MY, Kim DS, Kim SJ, Yoo HJ, et al. Generation of expandable human pluripotent stem cell-derived hepatocyte-like liver organoids. J Hepatol. 2019;71:970-85.

21. Ban HS, Han TS, Hur K, Cho HS. Epigenetic Alterations of Heat Shock Proteins (HSPs) in Cancer. Int J Mol Sci 2019, 20.

22. Cho HS, Han TS, Hur K, Ban HS. The Roles of Hypoxia-Inducible Factors and Non-Coding RNAs in Gastrointestinal Cancer. Genes (Basel) 2019, 10.

23. Han TS, Ban HS, Hur K, Cho HS. The Epigenetic Regulation of HCC Metastasis. Int J Mol Sci 2018, 19. 
24. Coppede F. The role of epigenetics in colorectal cancer. Expert Rev Gastroenterol Hepatol. 2014;8:935-48.

25. Okugawa Y, Grady WM, Goel A. Epigenetic Alterations in Colorectal Cancer: Emerging Biomarkers. Gastroenterology. 2015;149:1204-25 e1212.

26. Kim K, Ryu TY, Ryu JW, Han TS, Jung CR, Son MY, Kim DS, Cho HS. RNA-seq based transcriptome analysis of EHMT2 functions in breast cancer. Biochem Biophys Res Commun. 2020;524:672-6.

27. Kim K, Son MY, Jung CR, Kim DS, Cho HS. EHMT2 is a metastasis regulator in breast cancer. Biochem Biophys Res Commun. 2018;496:758-62.

28. Kim SK, Kim K, Ryu JW, Ryu TY, Lim JH, Oh JH, Min JK, Jung CR, Hamamoto R, Son MY, et al. The novel prognostic marker, EHMT2, is involved in cell proliferation via HSPD1 regulation in breast cancer. Int J Oncol. 2019;54:65-76.

29. Ryu TY, Kim K, Kim SK, Oh JH, Min JK, Jung CR, Son MY, Kim DS, Cho HS. SETDB1 regulates SMAD7 expression for breast cancer metastasis. BMB Rep. 2019;52:139-44.

30. Cho HS, Kelly JD, Hayami S, Toyokawa G, Takawa M, Yoshimatsu M, Tsunoda T, Field HI, Neal DE, Ponder BA, et al. Enhanced expression of EHMT2 is involved in the proliferation of cancer cells through negative regulation of SIAH1. Neoplasia. 2011;13:676-84.

31. Coon TA, McKelvey AC, Lear T, Rajbhandari S, Dunn SR, Connelly W, Zhao JY, Han S, Liu Y, Weathington NM, et al. The proinflammatory role of HECTD2 in innate immunity and experimental lung injury. Sci Transl Med. 2015;7:295ra109.

32. Tachibana M, Sugimoto K, Fukushima T, Shinkai Y. Set domain-containing protein, G9a, is a novel lysine-preferring mammalian histone methyltransferase with hyperactivity and specific selectivity to lysines 9 and 27 of histone H3. J Biol Chem. 2001;276:25309-17.

33. Tan ZW, Xie S, Hu SY, Liao T, Liu P, Peng KH, Yang XZ, He ZL, Tang HY, Cui Y, et al. Caudatin targets TNFAIP1/NF-kappaB and cytochrome c/caspase signaling to suppress tumor progression in human uterine cancer. Int J Oncol. 2016;49:1638-50.

34. Zhang M, Gao C, Yang Y, Li G, Dong J, Ai Y, Ma Q, Li W. MiR-424 Promotes Non-Small Cell Lung Cancer Progression and Metastasis through Regulating the Tumor Suppressor Gene TNFAIP1. Cell Physiol Biochem. 2017;42:211-21.

35. Zhang X, Li X, Tan Z, Liu X, Yang C, Ding X, Hu X, Zhou J, Xiang S, Zhou C, Zhang J. MicroRNA-373 is upregulated and targets TNFAIP1 in human gastric cancer, contributing to tumorigenesis. Oncol Lett. 2013;6:1427-34.

36. Zhou C, Li X, Zhang X, Liu X, Tan Z, Yang C, Zhang J. microRNA-372 maintains oncogene characteristics by targeting TNFAIP1 and affects NFkappaB signaling in human gastric carcinoma cells. Int J Oncol. 2013;42:635-42.

37. Cui J, Sun W, Hao X, Wei M, Su X, Zhang Y, Su L, Liu X. EHMT2 inhibitor BIX-01294 induces apoptosis through PMAIP1-USP9X-MCL1 axis in human bladder cancer cells. Cancer Cell Int. 2015;15:4.

38. Molineaux SM. Molecular pathways: targeting proteasomal protein degradation in cancer. Clin Cancer Res. 2012;18:15-20. 

Chain Fatty Acids as Key Bacterial Metabolites. Cell. 2016;165:1332-45.

\section{Figures}
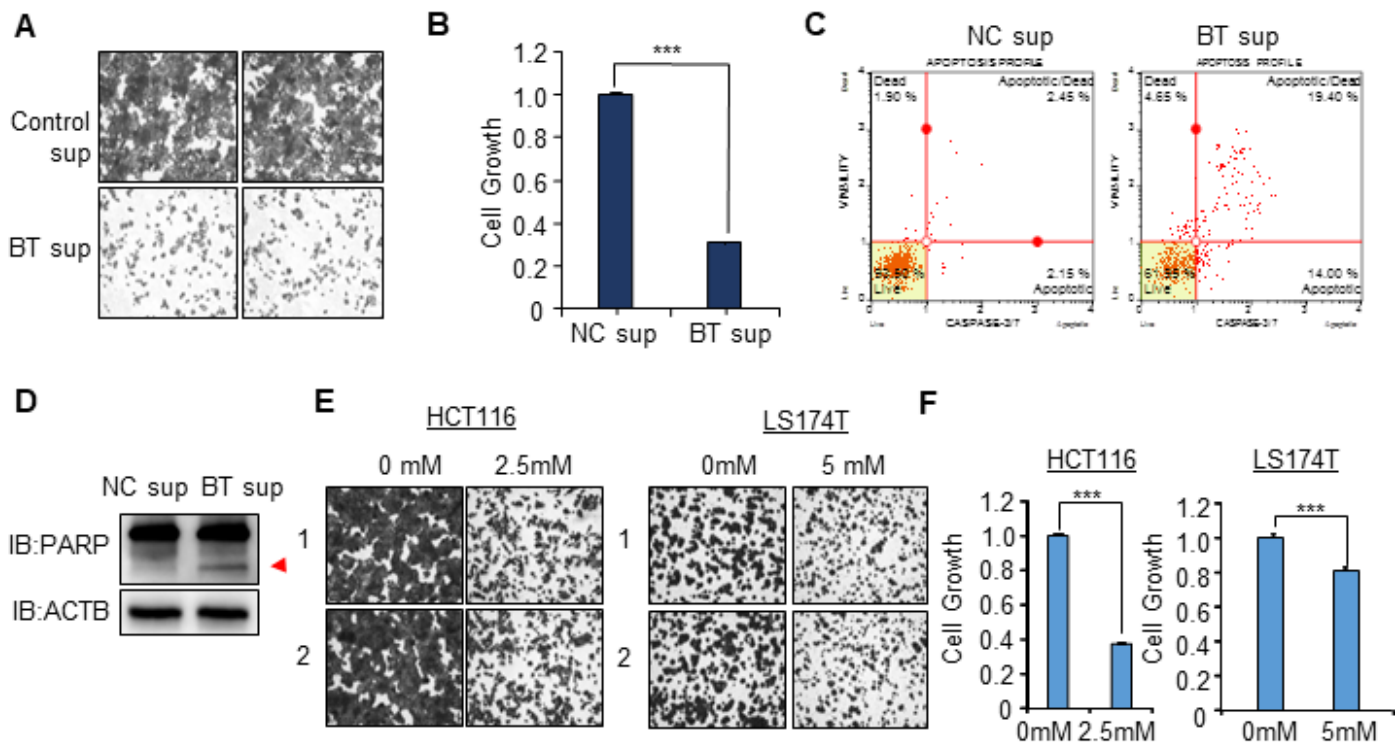

G

H

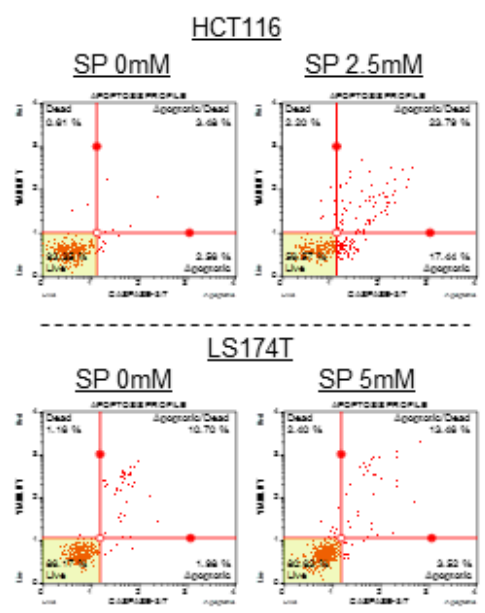

I
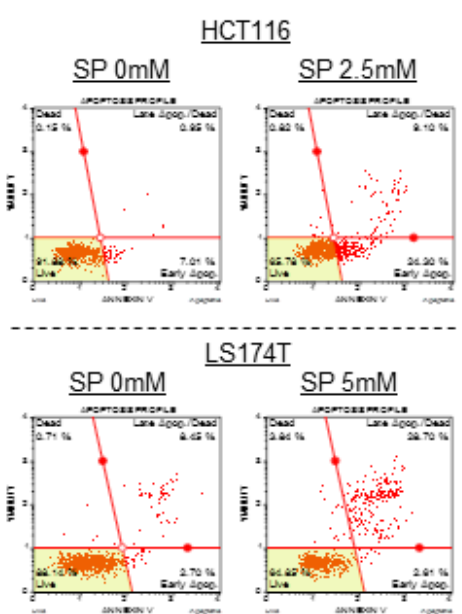

K

$J$

Gene Ontology (Biological Function)
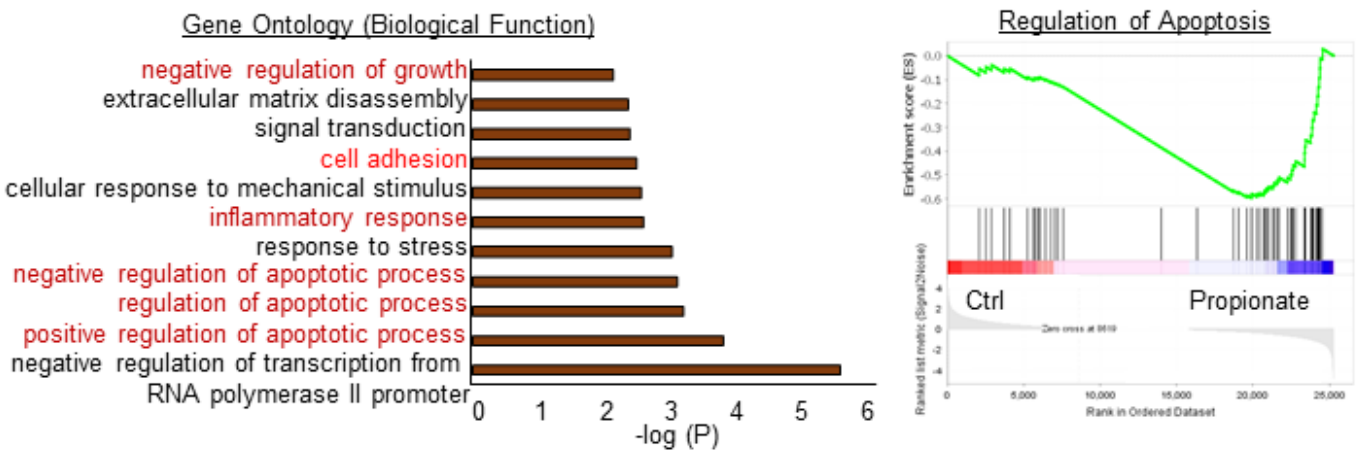

Figure 1.

\section{Figure 1}


The supernatant of BT culture medium and propionate suppressed the cell growth of HCT116 and LS174T cells via cell apoptosis. a, b Cell growth assay (crystal violet staining and CCK8 assay) after BT sup treatment for $48 \mathrm{~h}$. HCT116 cells were fixed in $100 \%$ methanol and stained with crystal violet solution (a). CCK-8 solution was added to the culture medium, and the cells were incubated for $5 \mathrm{~min}$ at $37^{\circ} \mathrm{C}$. The intensity of cell growth was measured by a microplate reader $(450 \mathrm{~nm})$. p values were calculated using Student's t-tests ( $* \star x p<0.001)(b)$. c FACS analysis using Muse Caspase-3/7 working solution was performed after treatment with BT sup. The upper right panel indicates the apoptotic and dead cell proportions. $d$ Western blot analysis after BT sup treatment using anti-PARP. ACTB was used as the internal control in HCT116 cells. e, f Cell growth assay after sodium propionate treatment for $48 \mathrm{~h}$. HCT116 and LS174T cells were fixed in 100\% methanol and stained with crystal violet solution (e). Cells were incubated for $5 \mathrm{~min}$ at $37^{\circ} \mathrm{C}$ after adding CCK-8 solution. The intensity of cell growth was measured by a microplate reader $(450 \mathrm{~nm})$. $p$ values were calculated using Student's t-tests $(* \star \star p<0.001)(\mathrm{f}) . \mathrm{g}$ Western blot analysis after sodium propionate treatment using anti-PARP. ACTB was used as the internal control in HCT116 and LS174T cells. h FACS analysis using Muse Caspase-3/7 working solution was performed after treatment with sodium propionate. The upper right panel indicates the apoptotic and dead cell proportions. i FACS analysis of Annexin V staining was carried out after treatment with sodium propionate. The lower right and upper right quadrants indicate early apoptosis and late apoptosis. j DAVID-based GO analysis of RNA-seq results with the up- and downregulated genes of the 872 genes. The enriched terms are shown. k GSEA analysis using 872 genes 
A

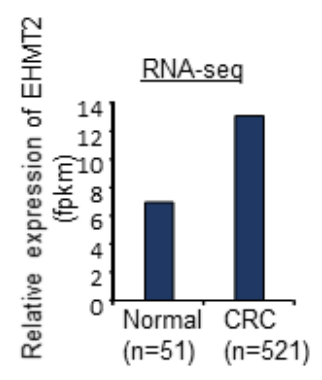

C

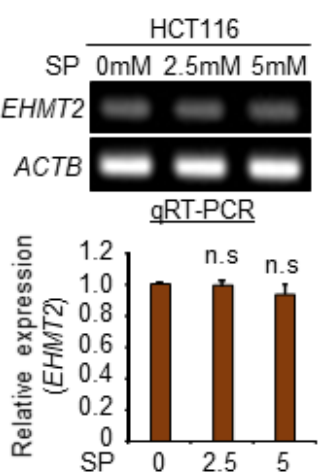

F

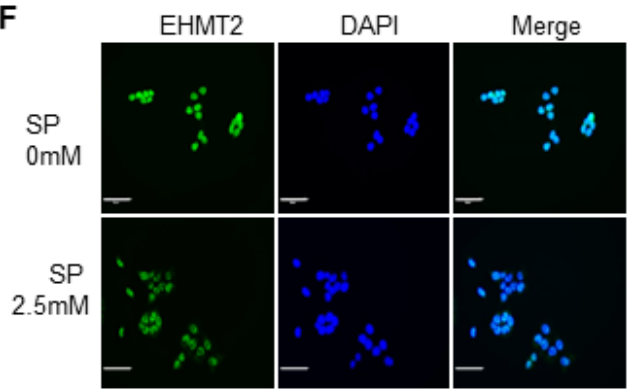

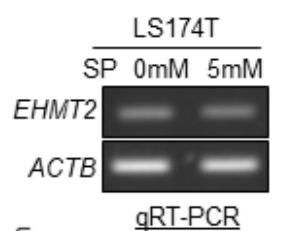

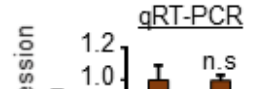

B

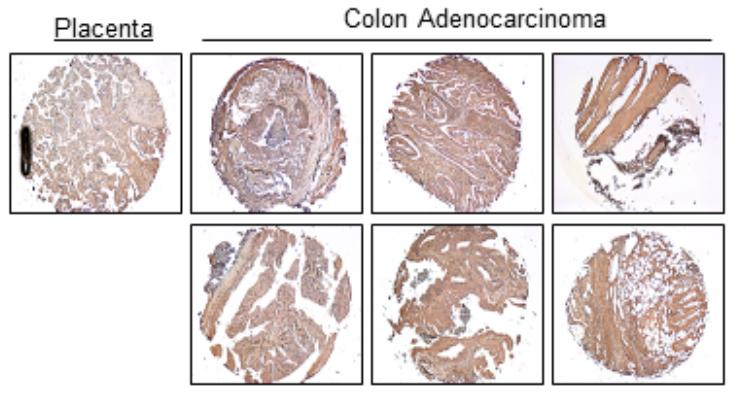

D

E
IB: EHMT2

IB: ACTB

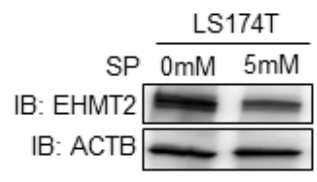

E

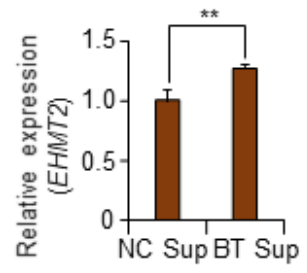

HCT116

NC Sup BT Sup

IB: EHMT2

IB: ACTB

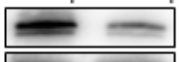

G

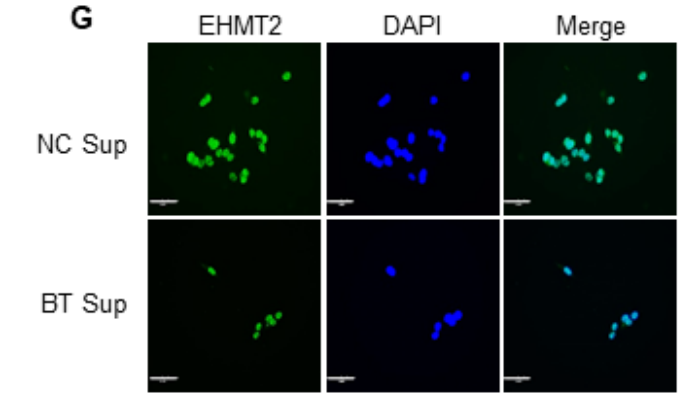

BT Sup

H

I $\mathrm{SP} \frac{0 \mathrm{~m} \mathrm{M}+\mathrm{CHX}}{0 \mathrm{~h} \quad 12 \mathrm{~h} 24 \mathrm{~h}} \frac{2.5 \mathrm{mM}+\mathrm{CHX}}{0 \mathrm{~h} \quad 12 \mathrm{~h} 24 \mathrm{~h}}$
IB:EHMT2
IB:ACTB

LS174T

IB: EHMT2 IB:ACTB
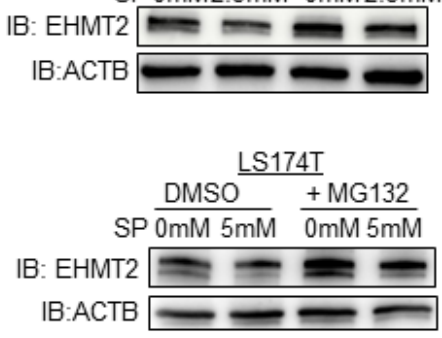

Figure 2

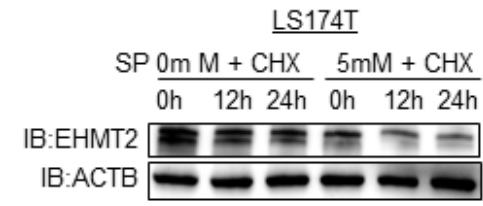

\section{Figure 2}

EHMT2 is a target for propionate-induced cell apoptosis in colon cancer. a The expression of EHMT2 in normal and CRC samples derived from TCGA portal. b Immunohistochemical analysis of EHMT2. A colon cancer tissue microarray was purchased from BioChain (https://www.biochain com). c The expression level of EHMT2 after treatment with sodium propionate in HCT116 and LS174T cells. RT-PCR analysis of EHMT2. Actin (ACTB) was used as an internal control (upper). qRT-PCR analysis of EHMT2 expression. p 
values were calculated using Student's t-tests (n.s; non-significant) (below). d Western blot analysis after sodium propionate treatment using anti-EHMT2. ACTB was used as the internal control in HCT116 and LS174T cells. e qRT-PCR analysis of EHMT2 expression after treatment with BT sup. p values were calculated using Student's t-tests ( $\left.{ }^{* *} \mathrm{p}<0.01\right)$ (upper). Western blot analysis after BT sup treatment using anti-EHMT2. ACTB was used as the internal control (below). $\mathrm{f}, \mathrm{g}$ Immunocytochemical analysis of EHMT2. HCT116 cells treated with sodium propionate (f) or BT sup $(\mathrm{g})$ were fixed with $100 \%$ methanol and stained with anti-EHMT2 (Alexa Fluor 488, green) and DAPI (blue). Scale bar, $50 \mu \mathrm{m}$. h Western blot analysis after treatment with SP for $24 \mathrm{~h}$ and MG132 for $6 \mathrm{~h}$ using anti-EHMT2. ACTB was used as the internal control in HCT116 and LS174T cells. i Western blot analysis of EHMT2 after treatment with SP for $24 \mathrm{~h}$ and cycloheximide (CHX) for $12 \mathrm{~h}$ and $24 \mathrm{~h}$. ACTB was used as the internal control. j SP induced ubiquitination of EHMT2. After transfection with a FLAG-tagged EHMT2 expression vector together with HA-tagged ubiquithin and treatment with SP for $24 \mathrm{~h}$ and MG132 for $6 \mathrm{~h}$. Western blot analysis was performed with HA and FALG antibodies. 
A

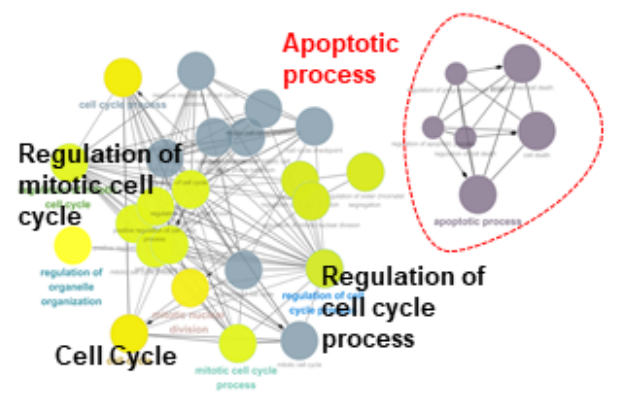

C
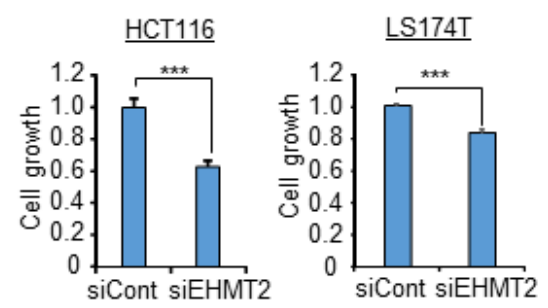

D

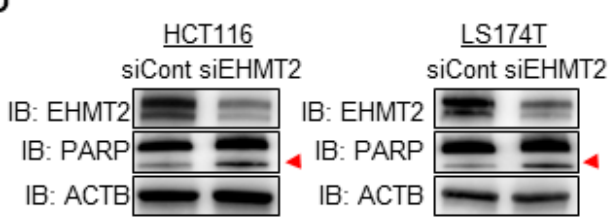

F

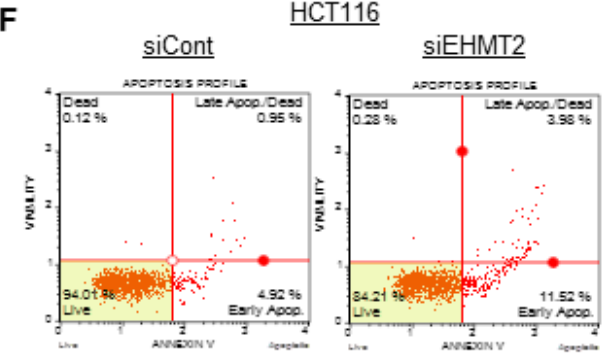

LS174T

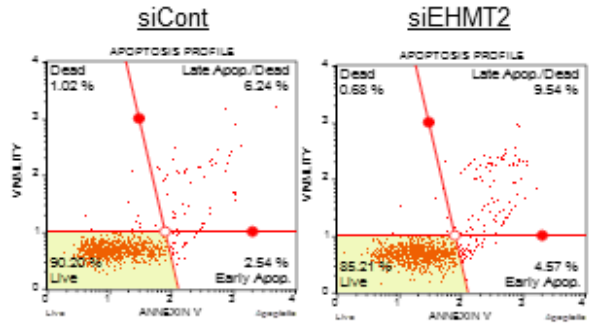

B
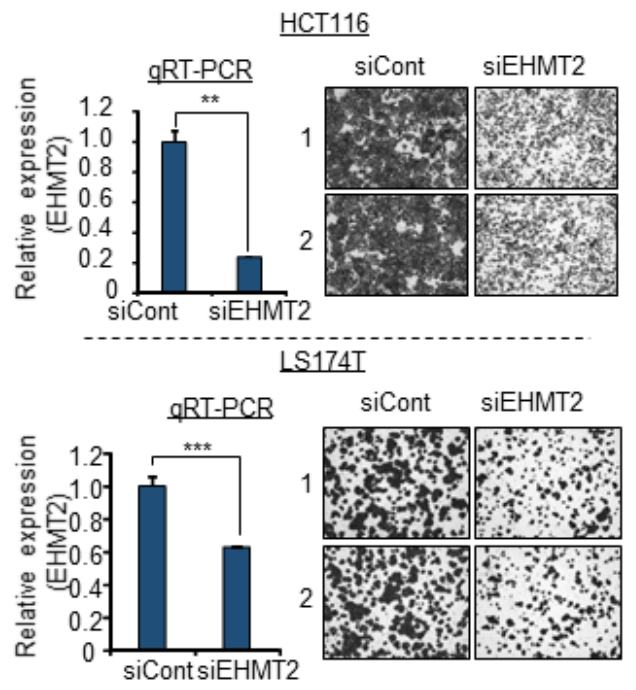

E

HCT116

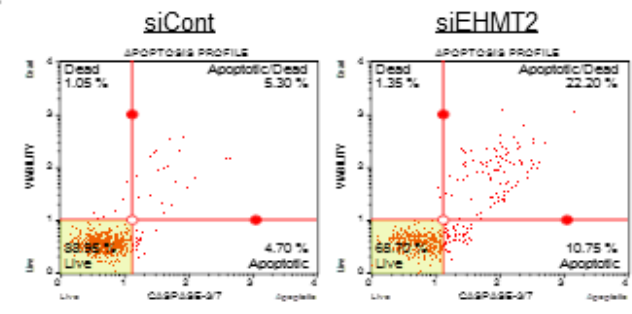

LS174T

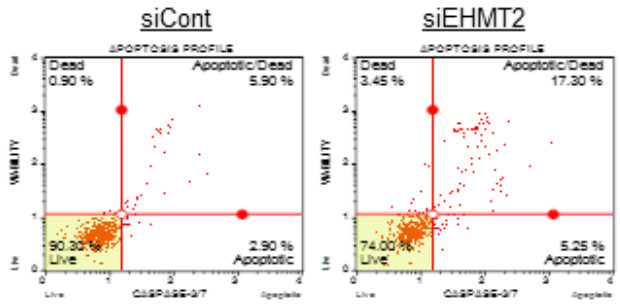

Figure 3

\section{Figure 3}

Knockdown of EHMT2 was involved in CRC apoptosis. a GO pathway term enrichment networks. GO pathway term networks in the EHMT2 knockdown and control groups were functionally grouped by ClueGO. Terms in the functionally grouped networks were cut off at $p$ values $>0.05$. b qRT-PCR analysis of EHMT2 after treatment with EHMT2 siRNA and siCont (negative control). p values were calculated using Student's t-tests (**p < 0.01) (left). Cell growth assay after treatment with siEHMT2 and siCont for 
48 h. HCT116 and LS174T cells were fixed in 100\% methanol and stained with crystal violet solution (right). c CCK-8 assay. CCK-8 solution was added to the culture medium after treatment with siEHMT2 and siCont, and the cells were incubated for $5 \mathrm{~min}$ at $37^{\circ} \mathrm{C}$. The intensity of cell growth was measured by a microplate reader $(450 \mathrm{~nm})$. $p$ values were calculated using Student's t-tests $(\star \star \star p<0.001)$. $d$ Western blot analysis after knockdown of EHMT2 using anti-EHMT2 and -PARP. ACTB was used as the internal control in HCT116 and LS174T cells. e FACS analysis using Muse Caspase-3/7 working solution was performed after EHMT2 knockdown. The upper right panel indicates the apoptotic and dead cell proportions. f FACS analysis of Annexin V staining was carried out after EHMT2 knockdown. The lower right and upper right quadrants indicate early apoptosis and late apoptosis. 
A

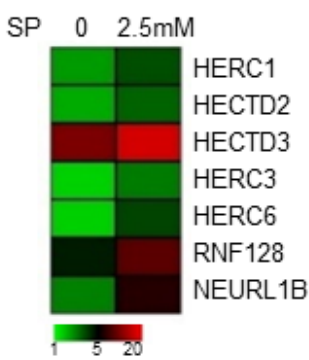

D

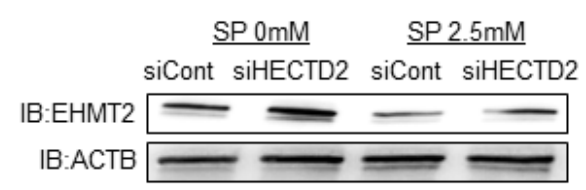

G
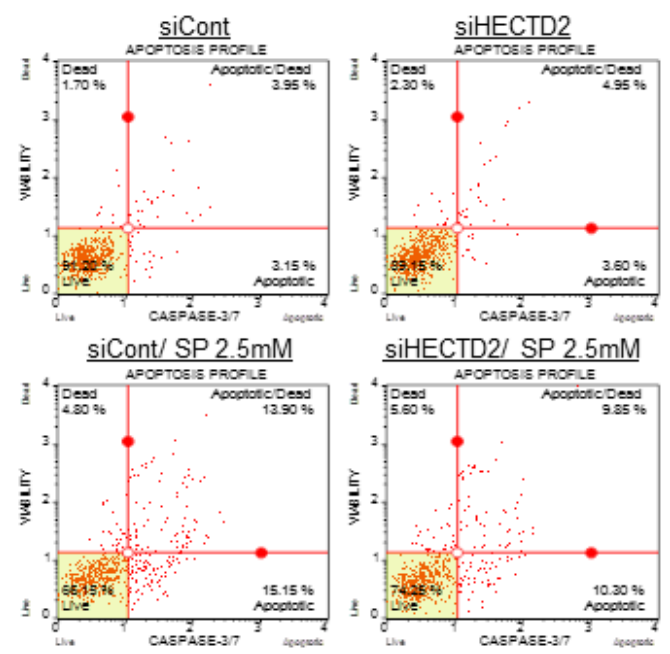

H

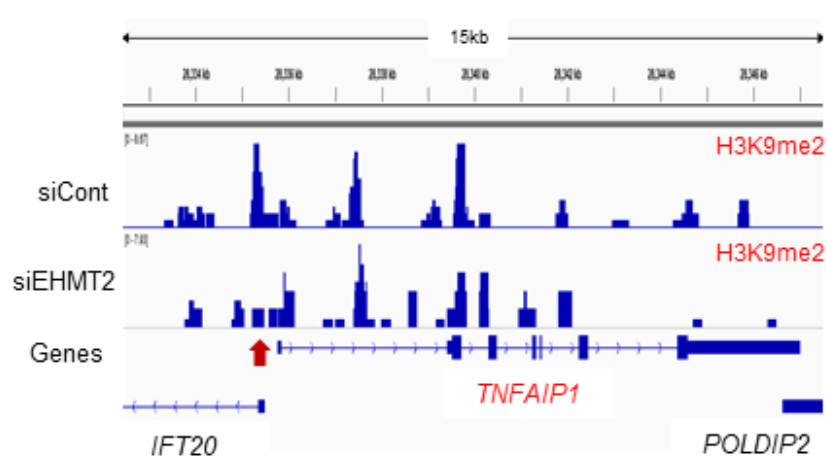

Figure 4.
C

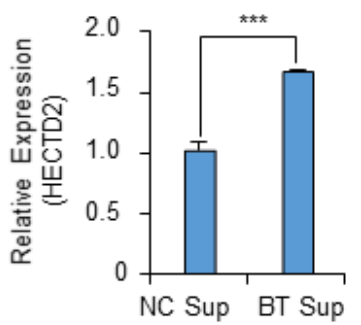

E SP $2.5 \mathrm{mM}$

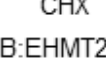

IB:ACTB

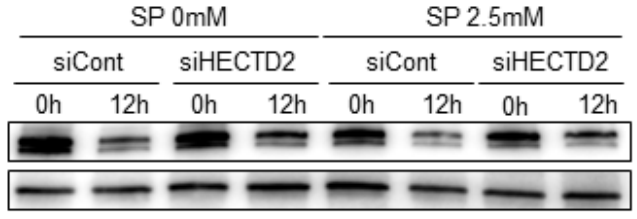

F

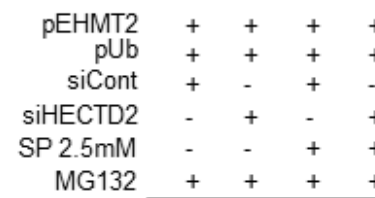

IP: FLAG IB: HA

IB: FLAG

IB: FLAG (Input)

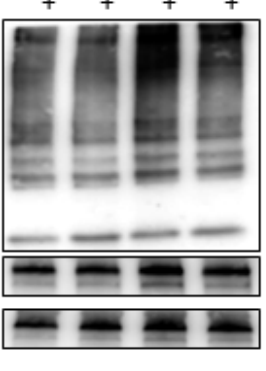

I

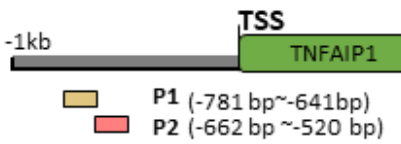

J

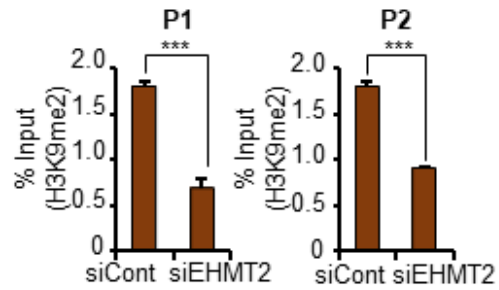

K

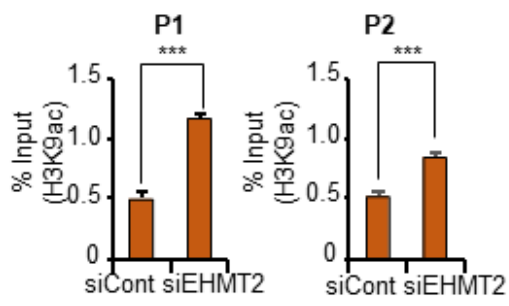

Figure 4

SP treatment induces HECTD2 expression for EHMT2 degradation a Heat map of RNA-seq data in the control and SP treatments. b, c qRT-PCR analysis of HECTD2 after treatment with SP (b; left) or BT sup (c; left). $p$ values were calculated using Student's t-tests (**p $<0.01$, $\left.{ }^{\star \star \star} p<0.001\right)$. $d$ Western blot analysis of EHMT2 after treatment with siHECTD2 and SP for $24 \mathrm{~h}$. ACTB was used as the internal control. e Western blot analysis of EHMT2 after treatment with siHECTD2 and SP for $24 \mathrm{~h}$ and CHX for $12 \mathrm{~h}$. ACTB was 
used as the internal control. $\mathrm{f}$ Western blot analysis after transfection with a FLAG-tagged EHMT2 expression vector together with HA-tagged ubiquitin and treatment with siHECTD2 and SP for $24 \mathrm{~h}$ and MG132 for $6 \mathrm{~h}$ using HA and FALG antibodies. g FACS analysis using Muse Caspase-3/7 working solution was performed after cotreatment with HECTD2 knockdown and SP in HCT116 cells. The upper right panel indicates the apoptotic and dead cell proportions. h ChIP-seq analysis. Representative IGV view of the H3K9me2 (siCont) and H3K9me2 (siEHMT2) ChIP signals in the promoter region of a TNFAIP1 gene. The promoter region was defined as the region from the transcription start site (TSS) to 1 $\mathrm{kb}$ upstream of the TSS. The Y-axis scale reflects the normalized number of reads using RPGC (number of reads per $10 \mathrm{bp} /$ scaling factor for $1 \mathrm{x}$ average coverage) of deepTools (v3.3.0). The normalized scales are 0 to 8.67 for H3K9me2 (siCont) and 0 to 7.93 for H3K9me2 (siEHMT2). i Graphical abstract for ChIP primer design on the TNFAIP1 promoter region. $\mathrm{j}, \mathrm{k}$ The ChIP assay was performed using anti-H3K9me2 (j) and anti-H3K9ac (k) antibodies. The result is shown as a percentage of input chromatin compared to the control in HCT116 cells after treatment with siEHMT2. P values were calculated using Student's t-test $(\star \star \star, P<0.001)$. 

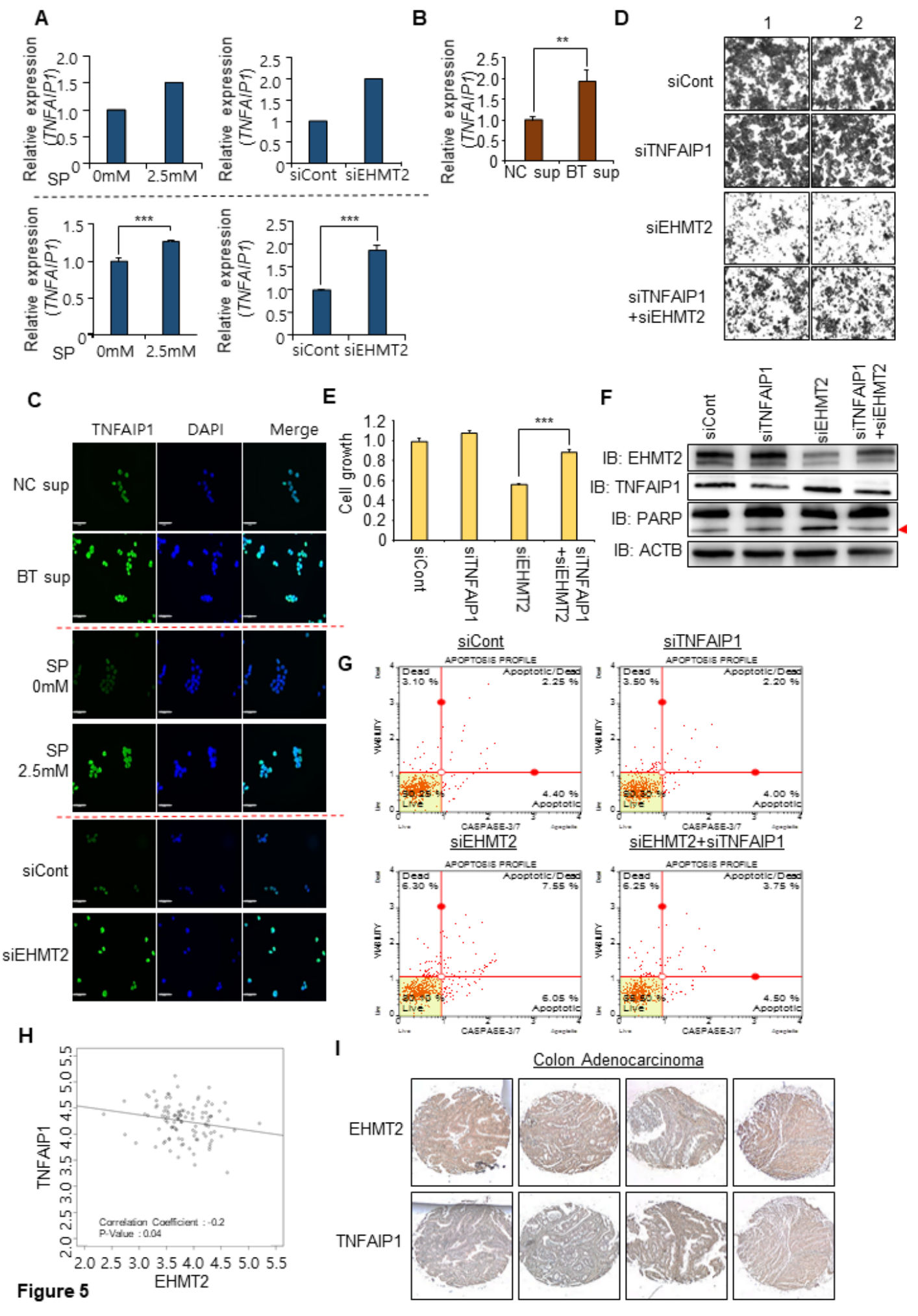

\section{Figure 5}

Negative regulation of TNFAIP1 by EHMT2 induced CRC apoptosis. a The expression level of TNFAIP1 in RNA-seq results after treatment with SP or siEHMT2 (upper). qRT-PCR analysis of TNFAIP1 after treatment with SP or siEHMT2. $p$ values were calculated using Student's t-tests ( $\left.{ }^{\star \star *} p<0.001\right)$ (below). $b$ qRT-PCR analysis of TNFAIP1 after treatment with BT sup. p values were calculated using Student's ttests (**p < 0.01). c Immunocytochemical analysis of TNFAIP1. HCT116 cells treated with SP or BT sup 
or siEHMT2 were fixed with $100 \%$ methanol and stained with anti-TNFAIP1 (Alexa Fluor 488, green) and DAPI (blue). Scale bar, $50 \mu \mathrm{m}$. d, e Cell growth assay after cotransfection with siTNFAIP1 and siEHMT2 for $48 \mathrm{~h}$. HCT116 cells were fixed in $100 \%$ methanol and stained with crystal violet solution (d). CCK-8 solution was added to the culture medium, and the cells were incubated for $5 \mathrm{~min}$ at $37^{\circ} \mathrm{C}$. The intensity of cell growth was measured by a microplate reader $(450 \mathrm{~nm})$. p values were calculated using Student's ttests $(* \star * p<0.001)(e)$. $f$ Western blot analysis after cotransfection of siEHMT2 and siTNFAIP1 using anti-PARP, -EHMT2, and -TNFAIP1 antibodies. ACTB was used as the internal control in HCT116 cells. $g$ FACS analysis using Muse Caspase-3/7 working solution was performed after cotransfection of siEHMT2 and siTNFAIP1. The upper right panel indicates the apoptotic and dead cell proportions. $\mathrm{h}$ Scatter plots of EHMT2 and TNFAIP1 in the GTEx portal. Each dotted line indicates a linear regression line of expression of EHMT2 and TNFAIP1. P values and correlation coefficients $(r)$ between two genes were obtained by the Pearson correlation method. i Immunohistochemical analysis of EHMT2 and TNFAIP1 in a colon cancer tissue microarray. 
A

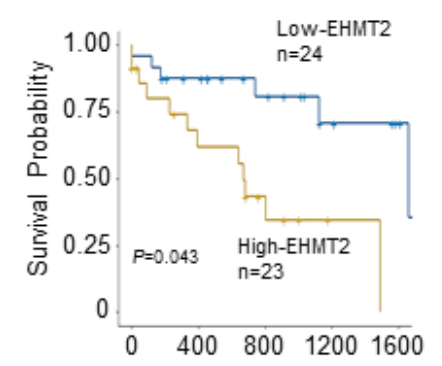

C

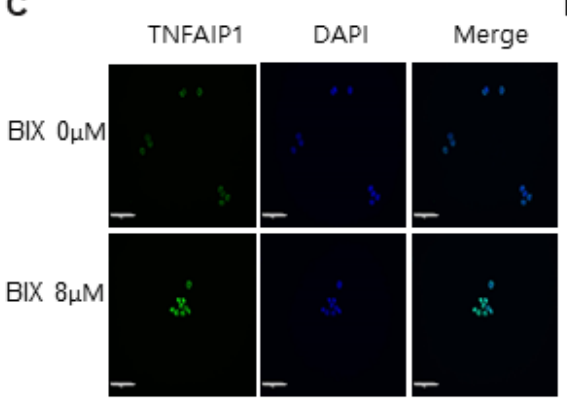

G

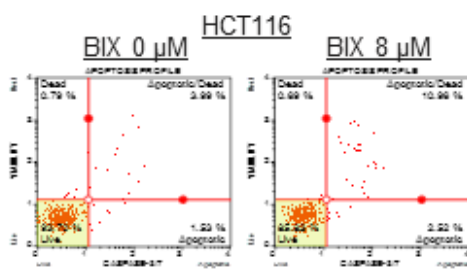

BIX $0 \mu M \quad$ LS174T BIX $10 \mu M$

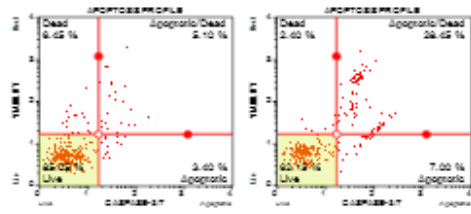

$\mathrm{H}$
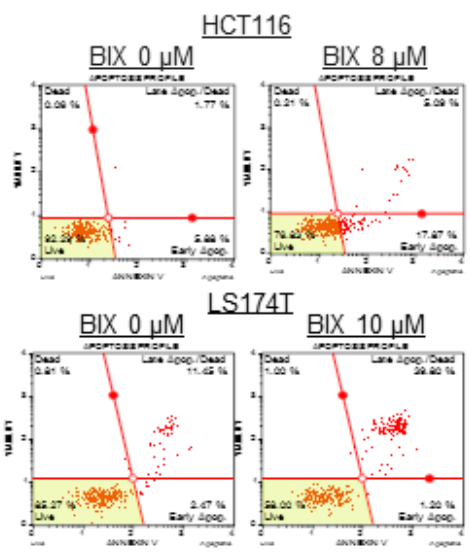

E
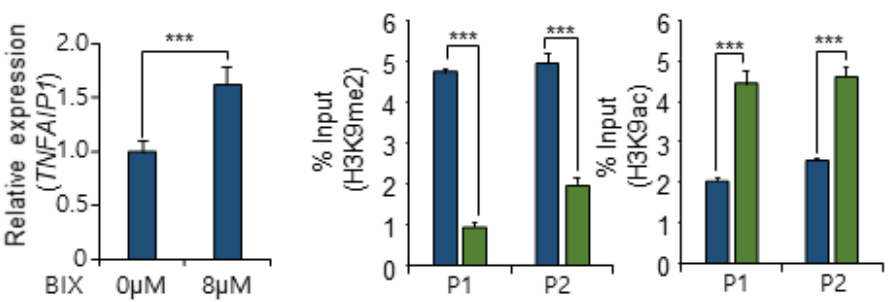

D

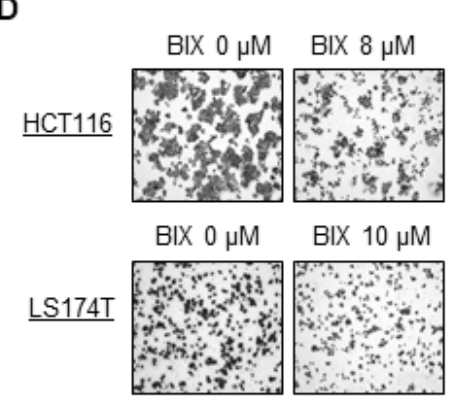

F

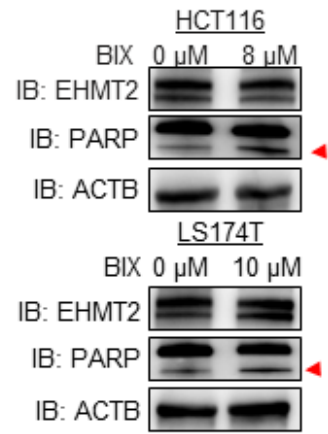

I
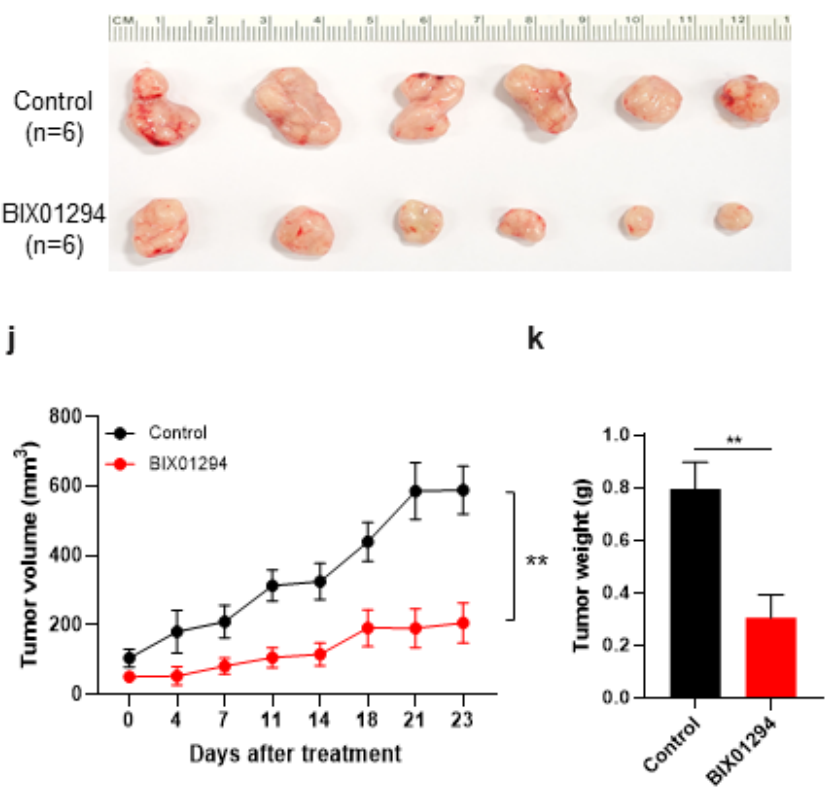

Figure 6

Figure 6

EHMT2 is a therapeutic target for CRC. a Kaplan-Meier plot depicting overall survival in CRC samples derived from the GTEx portal. The survival rate of the low EHMT2 group was significantly increased compared with that of the high EHMT2 group $(P=0.043)$. b qRT-PCR analysis of TNFAIP1 after treatment with BIX01294. $p$ values were calculated using Student's t-tests $(* \star * p<0.001)$. c Immunocytochemical analysis of TNFAIP1. HCT116 cells treated with BIX01294 were fixed with 100\% methanol and stained 
with anti-TNFAIP1 (Alexa Fluor 488, green) and DAPI (blue). Scale bar, $50 \mu \mathrm{m}$. d Cell growth assay after treatment with BIX01294 for $24 \mathrm{~h}$. HCT116 and LS174T cells were fixed in 100\% methanol and stained with crystal violet solution. e The ChIP assay was performed using anti-H3K9me2 (left) and anti-H3K9ac (right) antibodies. The result is shown as a percentage of input chromatin compared to the control in

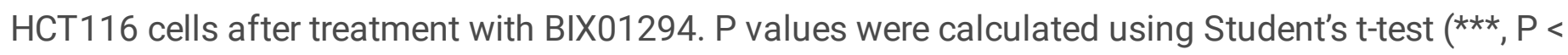
0.001). $f$ Western blot analysis after treatment with BIX01294 using anti-PARP and anti-EHMT2 antibodies. ACTB was used as the internal control in HCT116 and LS174T cells. g FACS analysis using Muse Caspase-3/7 working solution was performed after treatment with BIX01294. The upper right panel indicates the apoptotic and dead cell proportions. h FACS analysis of Annexin V staining was carried out after treatment with BIX01294. The lower right and upper right quadrants indicate early apoptosis and late apoptosis. i-k Treatment with BIX01294 suppressed xenograft nude mouse tumors. Either control or BIX01294 was intraperitoneally injected every three times a week after HCT116 cell implantation. (i) Macroscopic image of tumors on day 23, (j) tumor volumes, P values were calculated using 2-way ANOVA (***, $P<0.001)$ and $(k)$ tumor weight, $P$ values were calculated using Student's t-test $(* \star, P<0.01)$ 


\section{B. thetaiotaomicron}

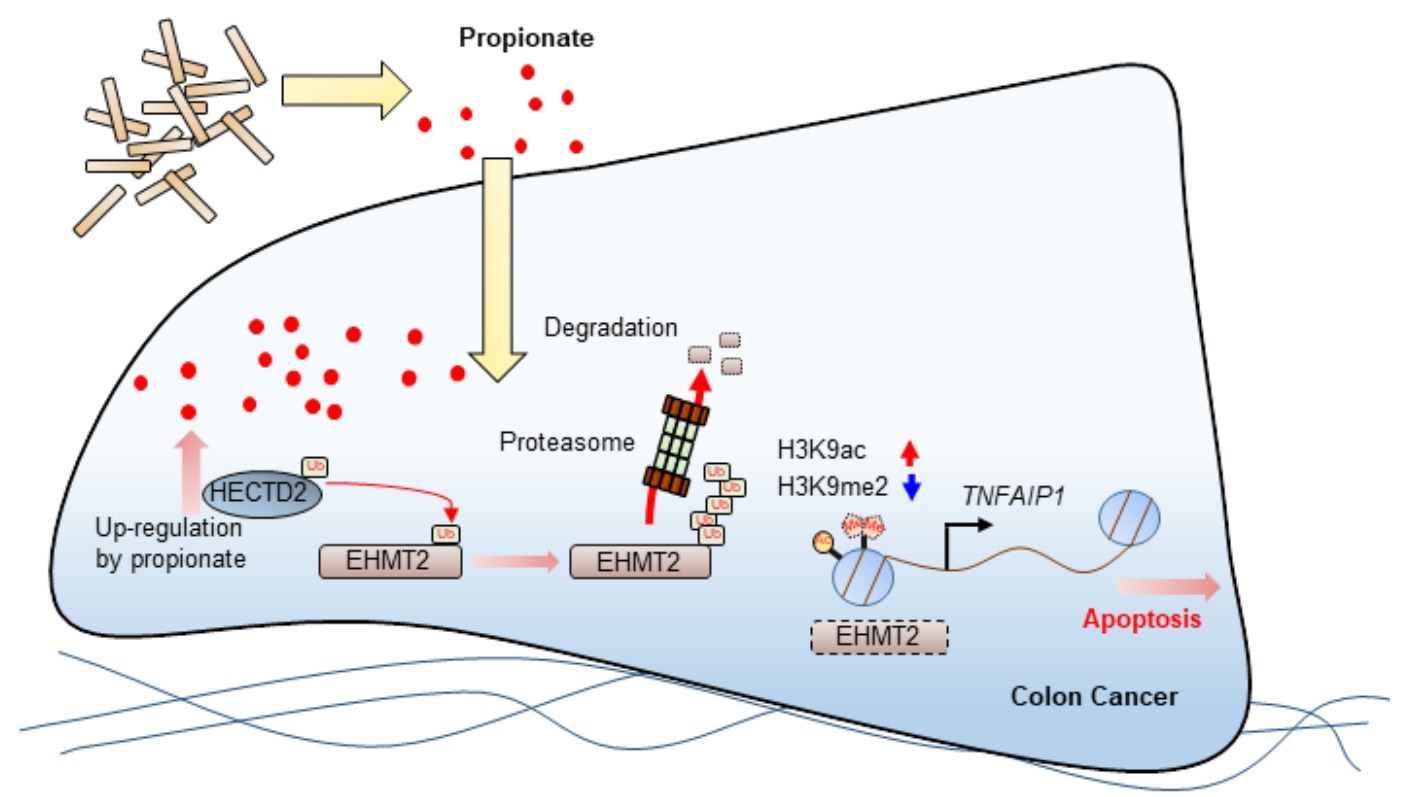

Figure 7

Figure 7

Schematic summary of BT-induced CRC apoptosis

\section{Supplementary Files}

This is a list of supplementary files associated with this preprint. Click to download. 
- EHMT2PropionateSupplementaryfigureV1.docx 